\title{
Pathological Brain Detection Using Weiner Filtering, 2D-Discrete Wavelet Transform, Probabilistic PCA, and Random Subspace Ensemble Classifier
}

\author{
Debesh Jha, ${ }^{1}$ Ji-In Kim, ${ }^{1}$ Moo-Rak Choi, ${ }^{2}$ and Goo-Rak Kwon' \\ ${ }^{1}$ Department of Information and Communication Engineering, Chosun University, 309 Pilmun-Daero, Dong-Gu, \\ Gwangju 61452, Republic of Korea \\ ${ }^{2}$ School of Electrical Engineering, Korea University, 145 Anam-ro, Sungbuk-gu, Seoul 02841, Republic of Korea
}

Correspondence should be addressed to Goo-Rak Kwon; grkwon@chosun.ac.kr

Received 10 May 2017; Revised 7 August 2017; Accepted 23 August 2017; Published 3 October 2017

Academic Editor: George A. Papakostas

Copyright (C) 2017 Debesh Jha et al. This is an open access article distributed under the Creative Commons Attribution License, which permits unrestricted use, distribution, and reproduction in any medium, provided the original work is properly cited.

\begin{abstract}
Accurate diagnosis of pathological brain images is important for patient care, particularly in the early phase of the disease. Although numerous studies have used machine-learning techniques for the computer-aided diagnosis (CAD) of pathological brain, previous methods encountered challenges in terms of the diagnostic efficiency owing to deficiencies in the choice of proper filtering techniques, neuroimaging biomarkers, and limited learning models. Magnetic resonance imaging (MRI) is capable of providing enhanced information regarding the soft tissues, and therefore MR images are included in the proposed approach. In this study, we propose a new model that includes Wiener filtering for noise reduction, 2D-discrete wavelet transform (2D-DWT) for feature extraction, probabilistic principal component analysis (PPCA) for dimensionality reduction, and a random subspace ensemble (RSE) classifier along with the $K$-nearest neighbors (KNN) algorithm as a base classifier to classify brain images as pathological or normal ones. The proposed methods provide a significant improvement in classification results when compared to other studies. Based on $5 \times 5$ cross-validation (CV), the proposed method outperforms 21 state-of-the-art algorithms in terms of classification accuracy, sensitivity, and specificity for all four datasets used in the study.
\end{abstract}

\section{Introduction}

Magnetic resonance imaging (MRI) of the brain provides comprehensive diagnostic information for diagnosis [1]. It is essential because it is noninvasive and safe and yields a higher resolution that cannot be obtained by other techniques. MRI is mainly utilized to diagnose different types of disorders such as strokes, tumors, bleeding, injury, blood-vessel diseases or infections, and multiple sclerosis (MS). The early diagnosis of pathological brain disease and its prodromal stage are critical and can decrease or halt the progression of the disease [2]. Therefore, the classification of normal/pathological brain status from MRIs is essential in clinical medicine as it focuses on soft tissue anatomy and generates a large and detailed dataset about the subject's brain. However, the use of a large database makes manual interpretation of the brain images tedious, time consuming, and costly. The major drawback of the manual approach is its irreducibility. Therefore, there is a need for automated image analysis tools such as computeraided diagnosis (CAD) systems [3].

Considerable research has been carried out to develop automatic tools for the classification of MR images to distinguish between normal and pathological brains. ElDahshan et al. [4] utilized a three-level discrete wavelet transform, accompanied by principal component analysis (PCA), to decrease features. A good success rate was obtained by using feedforward backpropagation neural networks (BPNNs) and the $K$-nearest neighbor (KNN). Zhang and $\mathrm{Wu}$ [5] recommended the application of a kernel support vector machine (KSVM) and presented three new kernels: homogenous polynomial, inhomogeneous polynomial, and Gaussian radial basis for distinguishing between normal 
and abnormal images. Patnaik et al. [6] employed DWT to obtain the approximation coefficients. Later, a support vector machine (SVM) was utilized to perform the classification. Zhang et al. [7] recommended a training feedforward neural network (FNN) with a unique scaled conjugate gradient (SCG) technique. Kundu et al. [8] proposed combining the Ripplet transform (RT) for feature extraction, PCA for dimensionality reduction, and the least-square SVM (LSSVM) for classification, and the $5 \times 5$ stratified crossvalidation (SCV) offered high classification accuracies. ElDahshan et al. [9] utilized the feedback pulse-coupled neural network for the preprocessing of MR images, the DWT for feature extraction, PCA for features reduction, and the FBPNN for the classification of pathological and normal brains. Damodharan and Raghavan [10] used wavelet entropy as the feature space, and they then used the traditional naiveBayes classifier classification method. Wang et al. [11] utilized the stationary wavelet transform (SWT) to substitute for DWT. Likewise, they proposed a hybridization of particle swarm optimization (PSO) and the artificial bee colony (HPA) method to obtain the optimal weights and biases of FNN. Nazir et al. [12] applied denoising at the beginning, and they achieved an overall classification accuracy of $91.8 \%$. Harikumar and Vinoth Kumar [13] used wavelet-energy and SVM. Padma and Sukanesh [14] used the combined wavelet statistical feature to segment and classify Alzheimer's disease (AD) as well as benign and malignant tumor slices. Zhang et al. [15] utilized Hu moment invariants (HMI) and generalized eigenvalue proximal SVM (GEPSVM) for the detection of pathological brain in MRI scanning and obtained an accuracy of $98.89 \%$, sensitivity of $99.29 \%$, and specificity of $92.00 \%$. Later on, Zhang et al. [16] used multilayer perceptron (MLP) for classification, where two pruning techniques like dynamic pruning (DP) and Bayesian detection boundaries (BDB were used to find the optimal hidden neurons and an adaptive real coded BBO (ARCBBO) method was implemented to determine the optimal weights and obtained an accuracy of $98.12 \%$ and $98.24 \%$, respectively. Nayak et al. [17] used 2DDWT, PCA, and Adaboost algorithm with random forest as its base classifier and obtained an accuracy of $98.44 \%$ for classification of pathological brain MR image with Dataset255. Later on, Nayak et al. [18] utilized two-dimensional stationary wavelet transform (SWT), symmetric uncertainty ranking (SUR) filter, and Adaboost with SVM classifier for the detection of pathological brain MR images and obtained an accuracy of $98.43 \%$ with Dataset-255. Wang et al. [19] employed Pseudo Zernike moment and linear regression classifier for classification of Alzheimer's disease and yielded an accuracy of $97.51 \%$, sensitivity of $96.71 \%$, and specificity of $97.73 \%$. Alam et al. [20] utilized dual-tree complex wavelet transform (DTCWT), principal component analysis (PCA), and twin support vector machine (TSVM) for the detection of Alzheimer's disease classification and obtained an accuracy of $95.46 \pm 1.26$.

Scholars have proposed different methods to extract features for the pathological brain disease [21]. After analyzing the above methods, we found that all of the methods achieved promising results which indicated that $2 \mathrm{D}-\mathrm{DWT}$ is effective in feature extraction for pathological brain detection.
However, there are two problems. (1) Most of them utilize traditional PCA for feature extraction which is computationalintensive for large datasets with a higher dimensions. (2) The classification performance can be further improved, because the feature vector contains excessive features, which required more memory and increased computational complexity. Moreover, it required too much time to train the classifiers.

To address the above-mentioned problems, we proposed a new pathological brain detection system based on brain MR images which has the potential improvements over the other schemes. Weiner filter is used for the preprocessing of the images. The proposed method uses 2D DWT for the extraction of features because of its ability to analyze images at different scales. PPCA is used in place of PCA for the reduction of features which has the advantages of computing the efficient dimension reduction in terms of the distribution of latent variables, maximum-likelihood estimates, probability model, dealing with the missing data, and a combination of multiple PCA as probabilistic mixture. A relatively new classifier known as random subspace ensemble (RSE) classifier is employed which has the advantage of low computational burden over the traditional classifiers. Hence, the novelty of the proposed method lies in the application of PPCA features and RSE classifier.

The article is organized as follows: Section 2 presents details about the materials and methods. Section 3 describes the experimental results, evaluation procedure, and discussions. Finally, Section 4 presents the conclusion and future research.

\section{Materials and Methods}

2.1. Materials. At present, there are four benchmark datasets (DS) as DS-66, DS-90, DS-160, and DS-255, of different sizes of $66,90,160$, and 255 images, respectively. All the datasets (DS) contain axial, T2-weighted, $256 \times 256$-pixel MR images downloaded from medical school of Harvard University (Boston, MA, USA) (URL: http://www.med.harvard.edu/ aablib/home.html) website. T2-weighted images are selected as input image because T2-weighted (spin-spin) relaxation gives better image contrast that is helpful to show different anatomical structure clearly. Also, they are better in detecting lesions than $\mathrm{T} 1$ weighted images.

We selected five slices from each subject. The selection criterion is that, for healthy subjects, these slices were selected at random. For pathological subjects, the slices should contain the lesions by confirmation of these radiologists with ten years of experiences. A sample of diseased slices is shown in Figure 2. In this investigation, all diseases are treated as pathological, and our task is a binary classification problem, that is, to distinguish pathological brain from healthy brains. Here, the whole brain is considered as the input image. We did not select local characteristics like point and edge, and we extract global image characteristics that are further learned by the new cascade model. Let us keep in mind that our procedure is different from the way neuroradiologists do. They usually take the local features and compare with standard template to check whether focuses exist, such as shrink, expansion, bleeding, and inflammation. While our technique 
is like AlphaGO, the computer researcher gives the machine sufficient data, and then the machine can learn how to make classification naturally. Including patients' information (age, gender, handedness, memory test, education, etc.) can add additional information and thus may assist us to improve the classification performance. Nevertheless, this new model proposed in our research is only dependent on the imaging data. Besides, the imaging data from the website does not contain the subjects' information.

The cost of predicting pathological to normal types is severe, because the subjects may be told that she/he is normal and thus avoids the mild symptoms displayed. The treatments of patients may be postponed. Nevertheless, the cost of misclassification of healthy to pathological types is low, since correct treatment can be given by other diagnosis means. The cost-sensitivity (CS) problem was resolved by changing the class distribution at the beginning state, since original data was accessible. That means we purposely picked up more pathological brains than healthy ones into the dataset, with the goal of making the classifier biased to pathological brains, to solve the CS problem. The overfitting problem was supervised by cross-validation technique.

In our experiment, DS-66 and DS-160 are extensively employed for brain MR image classifications that consist of normal brain images as well as abnormal brain images from seven types of diseases, namely, glioma, meningioma, Alzheimer's disease, Alzheimer's disease plus visual agnosia, Pick's disease, sarcoma, and Huntington's disease. DS-90 contains MR brain images of a healthy brain, AIDS dementia, Alzheimer's disease plus visual agnosia, Alzheimer's disease, cerebral calcinosis, cerebral toxoplasmosis, Creutzfeldt-Jakob disease, glioma, herpes encephalitis, Huntington's disease, Lyme encephalopathy, meningioma, metastatic adenocarcinoma, metastatic bronchogenic carcinoma, motor neuron disease, MS, Pick's disease, and sarcoma.

The third dataset, DS-255, includes images of four new types of diseases embedded with the above seven types of diseased images and normal brain images. The four additional diseases are chronic subdural hematoma, cerebral toxoplasmosis, herpes encephalitis, and MS.

2.2. Proposed Methodology. The proposed method comprises four vital stages, namely, image preprocessing, feature extraction using 2D-DWT, feature reduction utilizing PPCA, and classification using the RSE classifier. In order to enhance the quality of the MR images, Wiener filter is employed, followed by the extraction of approximation coefficients from MR images utilizing a 2D-DWT with three-level decomposition. Then, we saved these obtained features as our primary features. Thereafter, then we employ PPCA for obtaining uncorrelated discriminant set of features. Finally, we classified the reduced features using the RSE classifier with KNN as a base classifier. The complete block diagram of the proposed system is shown in Figure 1. A brief description about all these four stages is shown below.

2.2.1. Preprocessing Using Wiener Filter. The gif images were downloaded individually from the website of the Harvard Medical School. Then, each of the gif images was converted into JPG format manually. The images were in RGB format, and they were then converted into grayscale intensity images. Next, the intensity image is converted to double precision. Acquired brain MR images require preprocessing to improve the quality, enabling us to obtain better features. In our study, we used the popular Wiener filter method.

The Wiener filter is used to replace the finite impulse response (FIR) filter in order to decrease noise in signals [22]. When an image is blurred by a familiar low-pass filter (LPF), we can recover the image by inverse filtering. However, inverse filtering is extremely sensitive to additive noise. Wiener filtering accomplishes an optimal trade-off between inverse filtering and noise smoothing in that it eliminates the additive noise and inverts the blurring simultaneously. In addition, it reduces the overall mean-square error during the course of inverse filtering plus noise smoothing. The Wiener filtering method generates a linear approximation of the original image and is based on the stochastic framework. The orthogonality principle indicates that the Wiener filter in the Fourier domain can be articulated as follows:

$$
W\left(f_{1}, f_{2}\right)=\frac{H^{*}\left(f_{1}, f_{2}\right) S_{x x}\left(f_{1}, f_{2}\right)}{\left\|H\left(f_{1}, f_{2}\right)\right\|^{2} S_{x x}+S_{n n}\left(f_{1}, f_{2}\right)} .
$$

Here, $S_{x x}\left(f_{1}, f_{2}\right)$ is the power spectrum of the original image, $S_{n n}\left(f_{1}, f_{2}\right)$ is the adaptive noise, and $H\left(f_{1}, f_{2}\right)$ is the blurring filter.

\section{3. $2 D-D W T$}

2.3.1. Advantage of Wavelet Transform. The FT is the most commonly used tool for the analysis of signals, and it breaks down a time-domain signal into constituent sinusoids of various frequencies, thus changing the signal from the time domain to the frequency domain. Nevertheless, the FT has a serious disadvantage as it removes the time information from the signal. For instance, an investigator cannot determine when a specific event took place based on a Fourier spectrum. Therefore, the classification accuracy decreases as the time information is lost.

Gabor modified the FT to examine only a small part of the signal at a time. This approach is known as windowing or the short-time FT (STFT) [23]. It accumulates a window of appropriate shape to the signal. STFT can be considered as a compromise between the time information and frequency information. Nevertheless, the precision of the information is limited by the window size.

The wavelet transform (WT) constitutes the next logical step. It uses a windowing method with variable size, and the progress of the signal analysis is shown in Figure 3. Another benefit of the WT is that it selects a "scale" in place of the traditional "frequency"; that is, it does not generate a timefrequency view of a specific signal but a time-scale view. The time-scale view is another way of visualizing data and is more commonly used and effective.

2.3.2. DWT. This is an effective implementation of the WT, and it utilizes the dyadic scales and positions [24]. The 


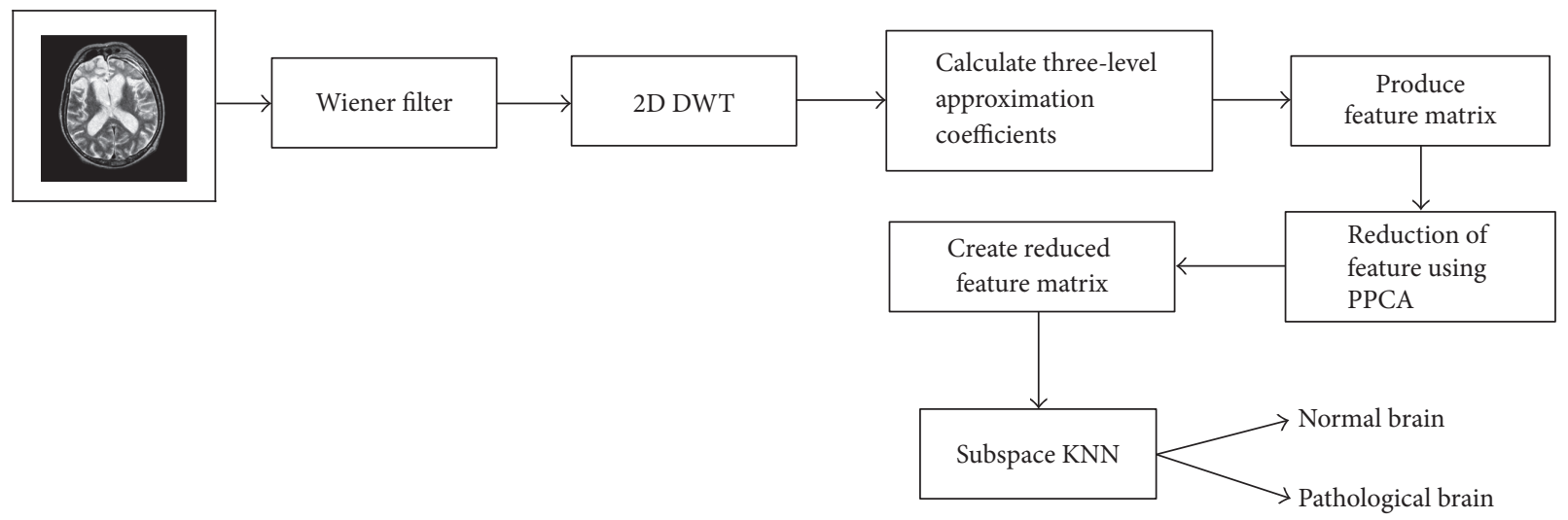

FIGURE 1: Block diagram of the proposed system.

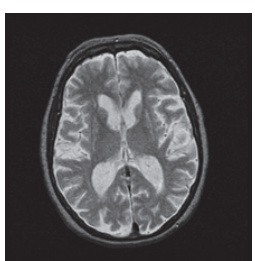

(a)

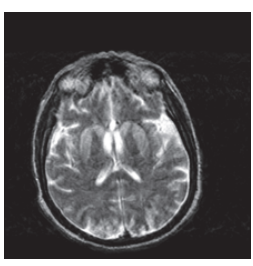

(g)

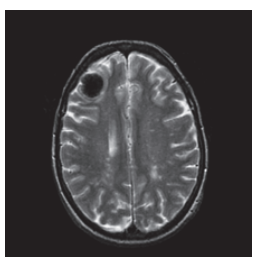

(m)

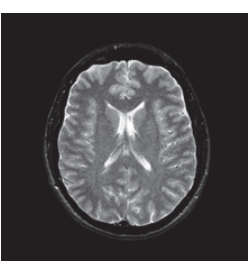

(b)

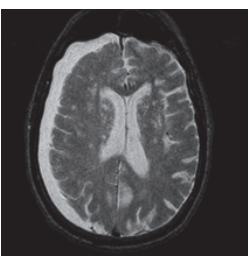

(h)

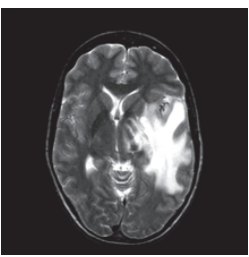

(n)

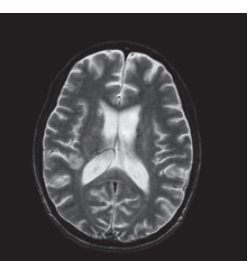

(c)

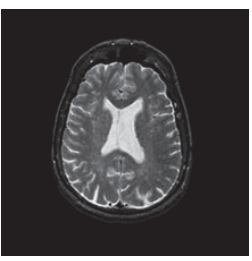

(i)

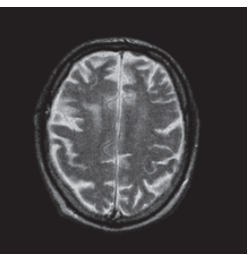

(o)

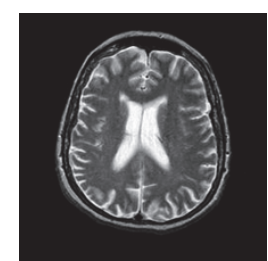

(d)

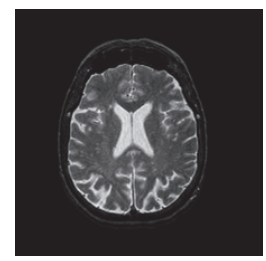

(j)

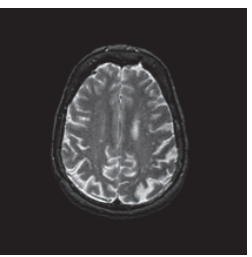

(p)

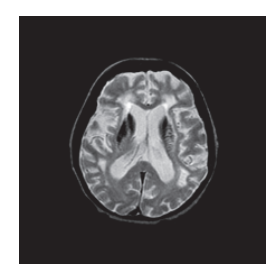

(e)

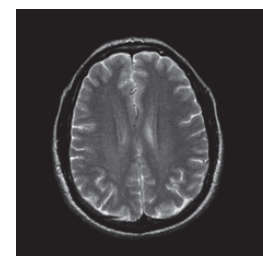

(k)

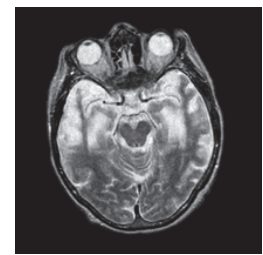

(q)

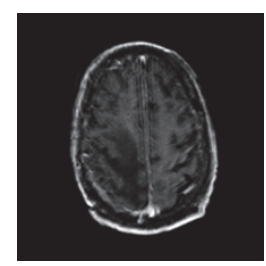

(f)

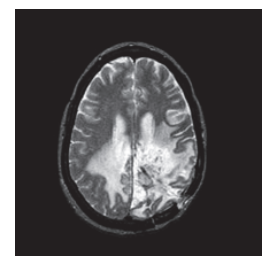

(l)

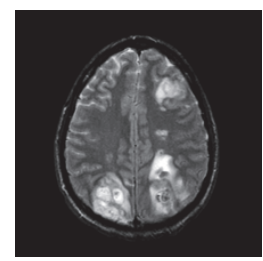

(r)

FIGURE 2: Brain MR images: (a) healthy brain; (b) AIDS dementia; (c) Alzheimer's disease plus visual agnosia; (d) Alzheimer's disease; (e) cerebral calcinosis; (f) cerebral toxoplasmosis; (g) Creutzfeldt-Jakob disease; (h) glioma, (i) herpes encephalitis; (j) Huntington's disease; (k) Lyme encephalopathy; (l) meningioma; (m) metastatic adenocarcinoma; (n) metastatic bronchogenic carcinoma; (o) motor neuron disease; (p) multiple sclerosis; (q) Pick's disease; and (r) sarcoma.

fundamentals of the DWT are as follows. Let $x(t)$ be a squareintegral function. The continuous WT of the signal $x(t)$ relative to a real-valued wavelet $\psi(\mathrm{t})$ is defined as

$$
W(a, \tau)=\int_{-\infty}^{\infty} x(t) \frac{1}{\sqrt{a}} \psi *\left(\frac{t-\tau}{a}\right) \mathrm{d} t,
$$

where $W(a, \tau)$ is the WT, $\tau$ indicates the function across $x(t)$, and the variable $a$ is the dilation factor (both real and positive numbers). Here, the asterisk $(*)$ indicates the complex conjugate.
Equation (1) can be discretized by restraining $a$ and $\tau$ to a discrete lattice $\left(a=2^{j}\right.$ and $\tau=2^{j} k$ ) to provide the DWT, which is given as follows:

$$
\begin{aligned}
& c A_{j, k}(n)=\operatorname{DS}\left[\sum_{n} x(n) l_{j}^{*}\left(n-2^{j} k\right)\right], \\
& c D_{j, k}(n)=\operatorname{DS}\left[\sum_{n} x(n) h_{j}^{*}\left(n-2^{j} k\right)\right] .
\end{aligned}
$$

Here, $c A_{j, k}$ and $c D_{j, k}$ refer to the coefficients of the approximation components and detailed components, 


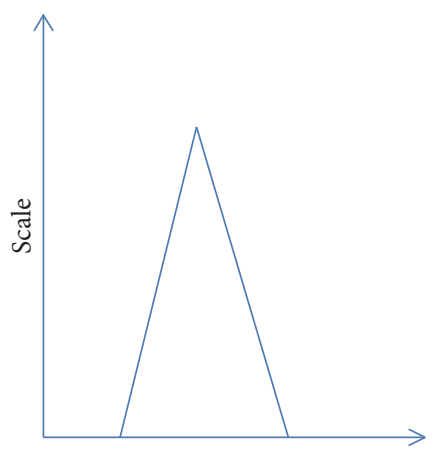

Frequency

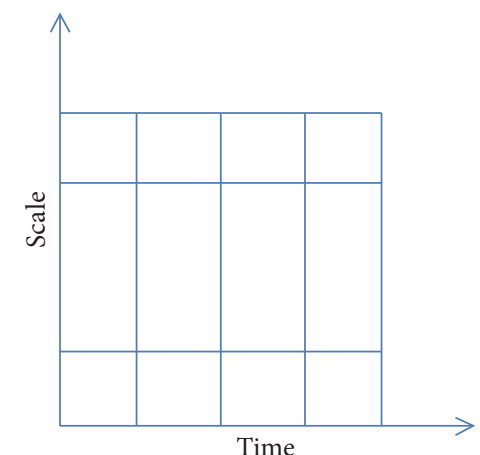

Time

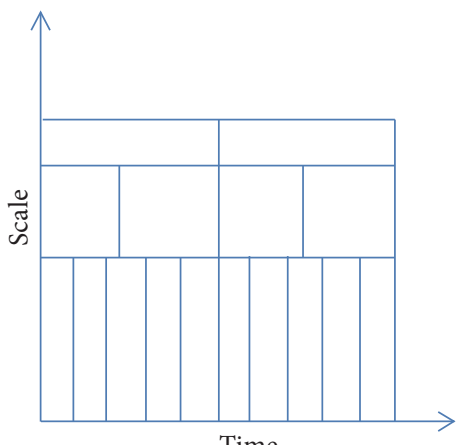

Time

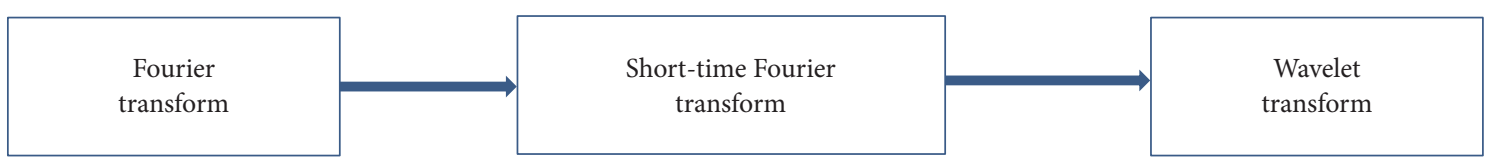

Figure 3: Progress of signal analysis.

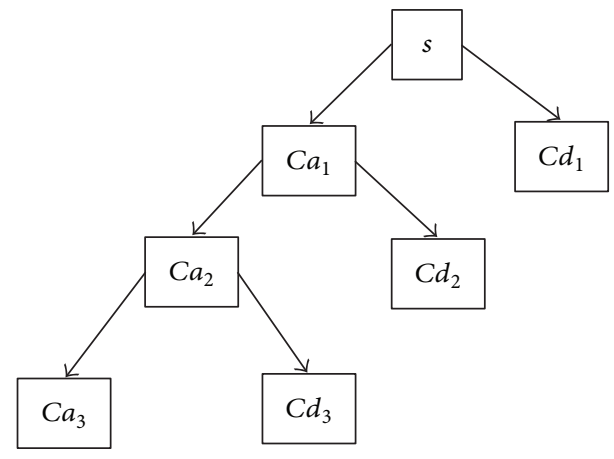

FIgURE 4: Three-level wavelet decomposition tree.

respectively. $l(n)$ and $h(n)$ represent the LPF and high-pass filter (HPF), respectively. $j$ and $k$ represent the wavelet scale and translation factors, respectively. The DS operator represents downsampling. The approximation component has low-frequency components of the image, whereas the detailed components contain high-frequency components. Figure 4 shows a three-level decomposition tree.

\subsection{3. $2 D-D W T$. In a case involving $2 \mathrm{D}$ images, the DWT} is employed in each dimension separately. A sample of a pathological brain MR image with its three-level wavelet decomposition is shown in Figure 5. Consequently, there are four subband images (LL, LH, HH, and HL) at each scale. The subband LL is utilized for the other 2D-DWT and can be considered as the approximation component of the image, whereas the LH, HL, and HH subbands can be considered as the detailed components of the image. As the level of the decomposition is increased, a more compact, but coarser approximation component is accessed. Thus, wavelets give a simple hierarchical foundation for clarifying the image information.

There are various types of wavelets, for example, Daubechies, symlets 1 , coiflets 1 , and biorthogonal wavelets and reverse biorthogonal 1.1. We tested our result with each type of the wavelet family as shown in Table 2. In our research, the approximation coefficient of three-level wavelet decomposition along with a Haar wavelet yields promising results when compared to others in the wavelet family. Hence, Haar wavelet was selected in the experiment. It is also the simplest and most significant wavelet of the wavelet family. Moreover, it is very fast and can be used to extract basic structural information from an image. All the features are present for all the images, and a feature matrix is generated.

2.4. Probabilistic Principal Component Analysis. The PPCA algorithm proposed by Tipping et al. [36-38] is based on the estimation of the principal axes when any input vector has one or more missing values. The PPCA reduces the high-dimensional data to a lower-dimensional representation by relating a $p$-dimensional observation vector $y$ to a $k$ dimensional latent (or unobserved) variable $x$ that is regarded as normal with zero mean and covariance $I(k)$. Moreover, PPCA depends on an isotropic error model. The relationship can be established as

$$
y^{T}=W * x^{T}+\mu+\varepsilon,
$$

where $y$ denotes the row vector of the observed variable, $\varepsilon$ denotes the isotropic error term, and $x$ is the row vector of latent variables. The error term, $\varepsilon$, is Gaussian with zero mean and covariance $v * I(k)$, where $v$ is the residual variance. To make the residual variance greater than 0 , the value of $k$ should be smaller than the rank. A standard principal component where $v$ equals 0 is the limiting condition of PPCA. The observed variables, $y$, are conditionally independent for the given values of the latent variables $x$. Therefore, the correlation between the observation variables is explained by the latent variables, and the error justifies the variability unique to $y_{i}$. The dimension of the matrix $W$ is $p \times k$, and it relates both latent and observation variables. The vector $\mu$ allows the model to acquire a nonzero mean. PPCA considers 


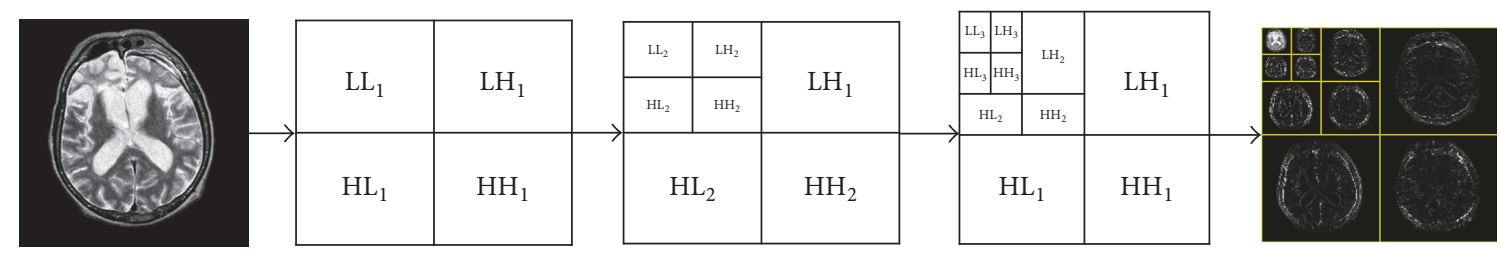

FIGURE 5: Pathological brain image and its wavelet coefficient at three-level decomposition.

the values to be missing and arbitrary over the dataset. From this model,

$$
y \sim N\left(\mu, W * W^{T}+v * I(k)\right)
$$

Given that the solution of $W$ and $v$ cannot be determined analytically, we used the expectation-maximization (EM) algorithm for the iterative maximization of the corresponding log-likelihood function. The EM algorithm considers missing values as additional latent variables. At convergence, the columns of $W$ span the solution subspace. PPCA then yields the orthonormal coefficients.

With respect to our research, the size of the image is 256 $\times$ 256. After three-level decomposition, the vector feature becomes $32 \times 32=1024$. Here, all the features are not relevant for the classification. Because of the high computational cost, we utilized PPCA for the dimensionality reduction. The advantage of PPCA over PCA is its computational efficiency.

2.5. RSE Classifier. Ensemble classification includes combining multiple classifiers to obtain more accurate predictions than those obtained utilizing individual models. In addition, ensemble learning techniques are considered very useful for upgrading prediction accuracy. Nevertheless, base classifiers must be as precise and diverse as possible to increase the generalization capability of an ensemble model.

For the classification of normal and pathological brain MRI images, we used a random subspace classifier that uses KNN as a base classifier. The main idea behind the success of ensemble classification is the diversification in the classification that makes the ensemble classifier. With the ensemble classification approach, each classifier provides a different error for different instant. Therefore, we can develop a strong classifier that can decrease the error. The random subspace classifier is a machine-learning classifier that divides the entire feature space into subspaces. Each subspace randomly selects features from the original feature space. It must be guaranteed that the boundaries of the particular base classifier are significantly different. To realize this, an unstable or weaker classifier is utilized as base classifier because they create sufficiently varied decision boundaries, even for small disturbances in the training data parameters.

We used the majority voting method to obtain the final decision of the class membership. In the proposed algorithm, we used KNN as the base classifier owing to its simplicity. After selecting a random subspace, a new set of KNNs is estimated. The majority voting method was utilized to combine the output of each base classifier for the decision preparing test class.
TABLE 1: Confusion matrix for a binary classifier to discriminate between two classes $\left(A_{1}\right.$ and $\left.A_{2}\right)$.

\begin{tabular}{lcc}
\hline \multirow{2}{*}{ True class } & \multicolumn{2}{c}{ Predicted class } \\
& $A_{1}$ (patients) & $A_{2}$ (controls) \\
\hline$A_{1}$ (patients) & $\mathrm{TP}$ & $\mathrm{FN}$ \\
$A_{2}$ (controls) & $\mathrm{FP}$ & $\mathrm{TN}$ \\
\hline
\end{tabular}

Here, TP (true positive): correctly categorized as positive cases, TN (true negative): correctly categorized as negative cases, FP (false positive): incorrectly categorized as negative cases, FN (false negative): incorrectly categorized as positive cases.

TABLE 2: Comparison of different wavelet families.

\begin{tabular}{lc}
\hline Wavelet family & Accuracy \\
\hline Haar & $99.20 \%$ \\
Daubechies 2 & $98.60 \%$ \\
Coiflets 1 & $96.98 \%$ \\
Symlets 1 & $99.01 \%$ \\
Biorthogonal 1.1 & $98.64 \%$ \\
\hline
\end{tabular}

2.6. Pseudocode of Proposed System. Our proposed system can be outlined in four major stages. The steps involved are depicted in Pseudocode 1.

2.7. Performance Measures. Various techniques are used to evaluate the classifier's efficiency. The performance is determined based on the final confusion matrix. The confusion matrix holds correct and incorrect classification results. Table 1 illustrates a confusion matrix for binary classification, where TP, TN, FP, and FN depict true positive, true negative, false positive, and false negative, respectively.

Here, pathological brains are assumed to hold the value "true," and normal control (NC) ones are assumed to hold the value "false" following normal convention. Now, we calculate the performance of the proposed approach on the basis of sensitivity, specificity, accuracy, and precision as follows.

(i) Sensitivity (true positive rate): this is the tendency or ability to determine that the diagnostic test is positive when the person has the disease:

$$
\text { Sensitivity }=\frac{\mathrm{TP}}{\mathrm{TP}+\mathrm{FN}} .
$$

(ii) Specificity (true negative rate): this is the tendency or ability to determine that the diagnostic test is negative when the person does not have the disease:

$$
\text { Specificity }=\frac{\mathrm{TN}}{\mathrm{TN}+\mathrm{FP}} \text {. }
$$


Input: T2-weighted MR brain images.

Parameter: $N$, total number of images

Step 1 (weiner filter)

for $i=1: N$

Read the images and apply wiener filter

end

Step 2 (2D-DWT)

For $i=1: N$

Read in the image file

Apply the DWT using for the 3rd level using "Haar" wavelet to extract the wavelet coefficients.

A matrix $X[M \times N]$ is employed to store all the coefficients.

End

Step 3. Reduce the features from the coefficients using PPCA

for $j=1: N$

Apply PPCA transformation on the obtained wavelet coefficients.

Put the new dataset in a matrix $Y$.

End

Step 4 (RSE classification using $5 \times 5$ cross-validation)

Divide the input data $I$ and target data $T$ into 5 different groups randomly

For $k=1: 5$

Use the $k$ th group for test, and other 4 groups to train the RSE algorithm.

Classify test image

End

Calculate average specificity, sensitivity, and accuracy.

Pseudocode 1: Pseudocode of the proposed system.

\begin{tabular}{|c|l|l|l|l|l|}
\hline Investigation 1 & & & & & \\
\hline Investigation 2 & & & & & \\
\hline Investigation 3 & & & & & \\
\hline Investigation 4 & & & & & \\
\hline Investigation 5 & & & & & \\
\hline
\end{tabular}

Training

Validation

FIGURE 6: Illustration of $k$-fold cross-validation.

(iii) Accuracy: this is a measure of how many diagnostic tests are correctly performed:

$$
\text { Accuracy }=\frac{\mathrm{TP}+\mathrm{TN}}{\mathrm{TP}+\mathrm{TN}+\mathrm{FP}+\mathrm{FN}} .
$$

(iv) The precision and the recall are formulated by

$$
\text { Precision }=\frac{\mathrm{TP}}{\mathrm{TP}+\mathrm{FP}} \text {. }
$$

2.8. Cross-Validation. Cross-validation (CV) is a modelassessment method that is used to evaluate the performance of a machine-learning algorithm prediction on a new DS on which it has not been trained. It helps to solve the overfitting problems. Each cross-validation round involves randomly portioning the original DS into a training set and a validation set. The illustration of the $k$-fold CV is shown in Figure 6 . The training set is used to train a supervised learning algorithm, while a test set is used to evaluate its performance.

To make the RSE classifier more reliable and generalize to independent datasets, a $5 \times 6$-fold stratified cross-validation $(\mathrm{SCV})$ and $5 \times 5$-fold SCV are employed. A $5 \times 6$-fold SCV is employed for DS-66 and $5 \times 5$-fold SCV is used for DS90, DS-160, and DS-255. For DS-66, 55 MR images are used for training whereas 75, 128, and 204 images are used for DS90 , DS-160, and DS-255 respectively. The validation images for DS-66, DS-90, DS-160, and DS-255 are 11, 15, 32, and 51, respectively. 
TABLE 3: Comparison result of the proposed method.

\begin{tabular}{lccccc}
\hline Proposed method & Feature & DS-66 & DS-90 & DS-160 & DS- 255 \\
\hline Logistic regression & 13 & 100.00 & 100.00 & 100.00 & 92.50 \\
QDA & 13 & 100.00 & 98.90 & 100.00 & 100.00 \\
KNN & 13 & 100.00 & 100.00 & 100.00 & 97.30 \\
RSE classifier & 13 & 100.00 & & 99.20 \\
\hline
\end{tabular}

TABLE 4: Classification comparison with DS-90.

\begin{tabular}{|c|c|c|c|c|c|}
\hline Existing methods & Success cases & Sensitivity (\%) & Specificity (\%) & Precision (\%) & Accuracy (\%) \\
\hline DWT + PCA + BPNN [25] & 388 & 88.00 & 56.00 & 97.14 & 86.22 \\
\hline $\mathrm{DWT}+\mathrm{PCA}+\mathrm{RBF}-\mathrm{NN}$ [25] & 411 & 92.47 & 72.00 & 98.25 & 91.33 \\
\hline $\mathrm{DWT}+\mathrm{PCA}+\mathrm{PSO}-\mathrm{KSVM}$ [25] & 440 & 98.12 & 92.00 & 99.52 & 97.78 \\
\hline $\mathrm{WE}+\mathrm{BPNN}[26]$ & 390 & 88.47 & 56.00 & 97.16 & 86.67 \\
\hline WE + KSVM [27] & 413 & 93.18 & 68.00 & 98.02 & 91.78 \\
\hline DWT + PCA + GA-KSVM [28] & 439 & 97.88 & 92.00 & 99.52 & 97.56 \\
\hline WE + PSO-KSVM [29] & 437 & 97.65 & 88.00 & 99.28 & 97.11 \\
\hline $\mathrm{WE}+\mathrm{BBO}-\mathrm{KSVM}[29]$ & 440 & 98.12 & 92.00 & 99.52 & 97.78 \\
\hline WE + QPSO-KSVM [30] & 442 & 98.59 & 92.00 & 99.52 & 98.22 \\
\hline WFRFT + PCA + GEPSVM [31] & 446 & 99.53 & 92.00 & 99.53 & 99.11 \\
\hline HMI + SEPSVM [15] & 445 & 99.06 & 96.00 & & 98.89 \\
\hline HMI + TSVM [15] & 445 & 99.29 & 92.00 & & 98.89 \\
\hline \multicolumn{6}{|l|}{ Proposed } \\
\hline 2D- DWT + PPCA + RSE (proposed) & 450 & 100.00 & 100.00 & 100.00 & 100.00 \\
\hline
\end{tabular}

\section{Results and Discussion}

In this study, we implemented a new machine-learning framework using MATLAB 2016a on an Intel computer with a Core-i5 processor and 16 GB RAM running under the Windows 7 operating system. This program can be tested or run on any computer platform where MATLAB is available.

3.1. Feature Extraction and Optimum Wavelet. In the proposed system, the three-level 2D-DWT of the Haar wavelet breaks down the input image into 10 subbands, as illustrated in Figure 5. The top left corner of the wavelet coefficient image (Figure 5) represents the approximation coefficients of the three-level decomposition of the image, whose size is only 32 $\times 32=1024$. These obtained features are the initial features. The size of these features is still large, and the matrix size needs to be reduced. Now, these reduced features are sent as the input to the PPCA.

3.2. Feature Reduction. The use of PPCA as a dimensionreduction tool reduces the feature size to its desired size. Here, we can take the feature as desired. It is better that the desired number of features should at least preserve more than $90 \%$ of the variance. However, in this study, we did not take $95 \%$ of the variance because it may lead to a higher computational cost. Researchers have considered different numbers of features. In our case, we first used a small number of features, but the accuracy was poor. However, the result with 13 principal components was excellent. Hence, the proposed method uses 13 principal components to earn higher classification accuracy.

3.3. Classification Results. The reduced features were sent to the classifier, and the results obtained with the different classifier are promising. From the experiment, it is seen that the proposed method works well for all four DSs using 13 principal components. The performances obtained with logistic regression, quadratic discriminant analysis, $\mathrm{KNN}$, and RSE classifier with KNN as a base classifier are shown in Table 3. From the table, we see that the proposed method outperforms other methods. We utilized a 5-fold CV for DS90, DS-160, and DS-255, whereas we utilized a 6-fold CV for DS-66. The RSE classifier obtained an accuracy of $100.00 \%$, $100.00 \%, 100.00 \%$, and $99.20 \%$, with DS-66, DS-90, DS-160, and DS-255, respectively. The result obtained with the cubic SVM is the same as the RSE classifier for the dataset beside DS-66, where it could only achieve $98.50 \%$.

3.4. Comparison with Existing Schemes. To further demonstrate the effectiveness of the proposed approach, we compared 21 existing algorithms. The algorithms and their corresponding results are listed in Tables 4 and 5. Table 4 shows the comparison result with DS-90. It is evident from Table 4 that our proposed method correctly matched all cases with $100 \%$ sensitivity, $100 \%$ specificity, $100 \%$ precision, and $100 \%$ accuracy. A comparison of the obtained results shows that our algorithm is superior to the others. This shows the effectiveness of the preprocessing technique combined with 
TABLE 5: Classification comparison (DS-66, DS-160, and DS-255).

\begin{tabular}{|c|c|c|c|c|c|}
\hline Approaches & Feature & Run & & ccuracy ( & \\
\hline DWT + SVM + POLY [24] & 4761 & & $D S-66$ & $D S-160$ & $D S-255$ \\
\hline DWT + SVM + RBF [24] & 4761 & 5 & 98.00 & 97.15 & 96.37 \\
\hline $\mathrm{DWT}+\mathrm{PCA}+k-\mathrm{NN}[4]$ & 7 & 5 & 98.00 & 97.33 & 96.18 \\
\hline $\mathrm{DWT}+\mathrm{PCA}+\mathrm{FNN}+\mathrm{ACPSO}[32]$ & 19 & 5 & 98.00 & 97.54 & 96.79 \\
\hline $\mathrm{DWT}+\mathrm{PCA}+\mathrm{FNN}+\mathrm{SCABC}[33]$ & 19 & 5 & 100.00 & 98.75 & 97.38 \\
\hline DWT + PCA + BPNN + SCG [7] & 19 & 5 & 100.00 & 98.93 & 97.81 \\
\hline DWT + PCA + KSVM [5] & 19 & 5 & 100.00 & 98.29 & 97.14 \\
\hline RT + PCA + LS-SVM [34] & 9 & 5 & 100.00 & 99.38 & 98.82 \\
\hline SWT + PCA + IABAP-FNN [11] & 7 & 10 & 100.00 & 98.88 & 98.43 \\
\hline $\mathrm{WT}+\mathrm{PCA}+\mathrm{ABC}-\mathrm{SPSO}-\mathrm{FNN}[11]$ & 7 & 10 & 100.00 & 99.44 & 99.18 \\
\hline $\mathrm{WE}+\mathrm{NBC}[35]$ & 7 & 10 & 92.58 & 99.62 & 99.02 \\
\hline DWT + PCA + ADBRF [17] & 13 & 5 & 100.00 & 99.30 & 98.44 \\
\hline DWT + SUR + ADBSVM [18] & 7 & 5 & 100.00 & 99.22 & 98.43 \\
\hline FRFE + DP-MLP + ARCBBO [16] & 12 & 10 & 100.00 & 99.19 & 98.24 \\
\hline FRFE + BDP-MLP + ARCBBO [16] & 12 & 10 & 100.00 & 99.31 & 98.12 \\
\hline $\mathrm{DWT}+\mathrm{PCA}+\mathrm{RSE}$ & 13 & 5 & 100.00 & 99.57 & 98.90 \\
\hline DWT + PPCA + RSE (proposed) & 13 & 5 & 100.00 & 100.00 & 99.20 \\
\hline
\end{tabular}

features extracted using the WT and PPCA. Table 4 shows the result of 5 runs of the proposed system. Table 5 demonstrates the comparison results over the three DSs in terms of the number of features, number of runs, and average accuracy. Here, some of the recent schemes were run 10 times, while others were run five times. From Tables 4 and 5, we see that most of the techniques achieved excellent classification when subjected to DS-66 as it is smaller in size. However, none of the algorithms achieved $100.00 \%$ with DS-90 and DS-160 because DS-255 is larger in size and includes more types of diseased brains; therefore, no current CAD system can earn a perfect classification.

Finally, this proposed "DWT + PPCA + RSE" achieved an accuracy of $100 \%$ for DS-66, DS-90, and DS-160 and an accuracy of $99.20 \%$ for DS-255, which is comparable with other recent studies and greater than the entire algorithm presented in Table 5. The improvement realized by the recommended scheme appears to be marginal compared with other schemes, but we obtained this result based on a careful statistical analysis (five repetitions of $k$-fold CV). Thus, this improvement is reliable and robust.

\section{Conclusion}

This paper proposed a new cascade model of " $2 \mathrm{D}-\mathrm{DWT}+$ PPCA + RSE" for the detection of pathological brains. The experiments validated its effectiveness as it achieved an accuracy of $99.20 \%$. Our contributions lie in three points. First, we introduced the Wiener filter and showed its effectiveness. Besides this we introduced the PPCA and RSE classifier and proved it gives the better performance when compared with other state-of-the-art algorithms. In this work, we transformed the PBD problem to a binary classification task. We presented a novel method that replaced PCA and introduced RSE classifier. The experiment showed the superiority of our methods to existing approaches.
The proposed algorithm can also be employed in other fields, for example, face recognition, breast cancer detection, and fault detection. Moreover, this method has been validated on the publically available datasets which are limited in size. Also, in the selected dataset, the images are collected during the late and middle stage of diseases; however, the images with disease at early stages need to be considered.

In future research, we may consider images from other modalities like MRSI, PET, and CT to increase robustness to our scheme. The proposed method can be validated on a larger clinical dataset utilizing modern machine-learning techniques like deep learning, extreme learning, and so on, after collecting the enough brain images from the medical institutes. Internet of things can be another promising research field to embed this PBDS.

\section{Nomenclature}

MR(I): Magnetic resonance (imaging)

DWT: Discrete wavelet transform

PPCA: Probabilistic principal component analysis

KNN: $\quad k$-nearest neighbor

$\mathrm{CV}$ : Cross-validation

BPNN: Backpropagation neural network

KSVM: Kernel support vector machine

SCG: $\quad$ Scale conjugate gradient

LS-SVM: Least-square support vector machine

FBPNN: Feedforward backpropagation neural network

SWT: $\quad$ Stationary wavelet transform

PSO: Particle swarm optimization

CAD: Computer-aided diagnosis

STFT: Short-time Fourier transform

QDA: Quadratic discriminant analysis

SUR: $\quad$ Symmetric uncertainty ranking 
PZM: $\quad$ Pseudo Zernike moment

SWT: Stationary wavelet transform

DTCWT: Dual-tree complex wavelet transform

RBFNN: Radial basis function neural network

CT: $\quad$ Computed tomography

TSVM: Twin support vector machine

HMI: Hu moment invariants

MLP: $\quad$ Multilayer perceptron

ARCBBO: Adaptive real coded biogeography-based optimization

DP: $\quad$ Dynamic pruning.

\section{Conflicts of Interest}

The authors declare that they have no conflicts of interest.

\section{Acknowledgments}

This research was supported by the Brain Research Program through the National Research Foundation of Korea funded by the Ministry of Science, ICT \& Future Planning (NRF2014M3C7A1046050). And this work was supported by the National Research Foundation of Korea Grant funded by the Korean Government (NRF-2017R1A2B4006533).

\section{References}

[1] D. Jha and G.-R. Kwon, "Alzheimers disease detection in MRI using curvelet transform with K-NN," Journal of KIIT, vol. 14, no. 8, 2016.

[2] S. Alam, M. Kang, J.-Y. Pyun, and G.-R. Kwon, "Performance of classification based on PCA, linear SVM, and Multi-kernel SVM," in Proceedings of the 8th International Conference on Ubiquitous and Future Networks, ICUFN 2016, pp. 987-989, Vienna, Austria, July 2016.

[3] F. Thorsen, B. Fite, L. M. Mahakian et al., "Multimodal imaging enables early detection and characterization of changes in tumor permeability of brain metastases," Journal of Controlled Release, vol. 172, no. 3, pp. 812-822, 2013.

[4] E.-S. A. El-Dahshan, T. Hosny, and A.-B. M. Salem, "Hybrid intelligent techniques for MRI brain images classification," Digital Signal Processing, vol. 20, no. 2, pp. 433-441, 2010.

[5] Y. Zhang and L. Wu, "An MR brain images classifier via principal component analysis and kernel support vector machine," Progress in Electromagnetics Research, vol. 130, pp. 369-388, 2012.

[6] L. M. Patnaik, S. Chaplot, and N. R. Jagannathan, "Classification of magnetic resonance brain images using wavelets as input to support vector machine and neural network," Biomedical Signal Processing and Control, vol. 1, no. 1, pp. 86-92, 2006.

[7] Y. Zhang, Z. Dong, L. Wu, and S. Wang, "A hybrid method for mri brain image classification," Expert Systems with Applications, vol. 38, no. 8, pp. 10049-10053, 2001.

[8] M. K. Kundu, M. Chowdhury, and S. Das, "Brain MR image classification using multi-scale geometric analysis of ripplet," Progress in Electromagnetics Research, vol. 137, pp. 1-17, 2013.

[9] E. A.-S. El-Dahshan, H. M. Mohsen, K. Revett, and A.-B. M. Salem, "Computer-aided diagnosis of human brain tumor through MRI: A survey and a new algorithm," Expert Systems with Applications, vol. 41, no. 11, pp. 5526-5545, 2014.
[10] S. Damodharan and D. Raghavan, "Combining tissue segementation and neural network for brain tumor detection," The International Arab Journal of Information Technology, vol. 12, no. $1,2015$.

[11] S. Wang, Y. Zhang, Z. Dong et al., "Feed-forward neural network optimized by hybridization of PSO and ABC for abnormal brain detection," International Journal of Imaging Systems and Technology, vol. 25, no. 2, pp. 153-164, 2015.

[12] M. Nazir, F. Wahid, and S. A. Khan, "A simple and intelligent approach for brain MRI classification," Journal of Intelligent \& Fuzzy Systems. Applications in Engineering and Technology, vol. 28, no. 3, pp. 1127-1135, 2015.

[13] R. Harikumar and B. Vinoth Kumar, "Performance analysis of neural networks for classification of medical images with wavelets as a feature extractor," International Journal of Imaging Systems and Technology, vol. 25, no. 1, pp. 33-40, 2015.

[14] A. Padma and R. Sukanesh, "Segementation and classification of brain CT images using combined wavelet statistical texture features," Arabian Journal for Science Engineering, vol. 39, no. 2, 2014.

[15] Y. Zhang, J. Yang, S. Wang, Z. Dong, and P. Phillips, "Pathological brain detection in MRI scanning via Hu moment invariants and machine learning," Journal of Experimental and Theoretical Artificial Intelligence, vol. 29, no. 2, pp. 299-312, 2017.

[16] Y. Zhang, Y. Sun, P. Phillips, G. Liu, X. Zhou, and S. Wang, "A multilayer perceptron based smart pathological brain detection system by fractional fourier entropy," Journal of Medical Systems, vol. 40, no. 7, article 173, 2016.

[17] D. R. Nayak, R. Dash, and B. Majhi, "Brain MR image classification using two-dimensional discrete wavelet transform and AdaBoost with random forests," Neurocomputing, vol. 177, pp. 188-197, 2016.

[18] D. R. Nayak, R. Dash, and B. Majhi, "Stationary wavelet transform and AdaBoost with SVM based pathological brain detection in MRI scanning," CNS and Neurological Disorders Drug Targets, vol. 16, no. 2, pp. 137-149, 2017.

[19] S.-H. Wang, S. Du, Y. Zhang et al., "Alzheiemers disease detection by pseudo zernike moment and linear regression classifier," CNS \& Neurologicla Disorders, vol. 16, no. 1, pp. 1115, 2017.

[20] S. Alam, M. Kang, and G. Kwon, "Alzheimer disease classification based on TSVM and Kernel SVM," in Proceedings of the 2017 Ninth International Conference on Ubiquitous and Future Networks (ICUFN), pp. 565-567, July 2017.

[21] D. Jha, J. Kim, and G. Kwon, "Diagnosis of Alzheimer's disease using dual-tree complex wavelet transform, PCA, and feedforward neural network," Journal of Healthcare Engineering, vol. 2017, Article ID 9060124, 13 pages, 2017.

[22] H. Naimi, A. B. H. Adamou-Mitiche, and L. Mitiche, "Medical image denoising using dual tree complex thresholding wavelet transform and Wiener filter," Journal of King Saud University - Computer and Information Sciences, vol. 27, no. 1, pp. 40-45, 2015.

[23] L. Durak, "Shift-invariance of short-time FOUrier transform in fractional FOUrier domains," Journal of the Franklin Institute. Engineering and Applied Mathematics, vol. 346, no. 2, pp. 136146, 2009.

[24] S. Chaplot, L. M. Patnaik, and N. R. Jagannathan, "Classification of magnetic resonance brain images using wavelets as input to support vector machine and neural network," Biomedical Signal Processing and Control, vol. 1, no. 1, pp. 86-92, 2006. 
[25] Y. Zhang, S. Wang, G. Ji, and Z. Dong, "An MR brain images classifier system via particle swarm optimization and kernel support vector machine," The Scientific World Journal, vol. 2013, Article ID 130134, 9 pages, 2013.

[26] R. Choudhary, S. Mahesh, J. Paliwal, and D. S. Jayas, "Identification of wheat classes using wavelet features from near infrared hyperspectral images of bulk samples," Biosystems Engineering, vol. 102, no. 2, pp. 115-127, 2009.

[27] M. R. K. Mookiah, U. Rajendra Acharya, C. M. Lim, A. Petznick, and J. S. Suri, "Data mining technique for automated diagnosis of glaucoma using higher order spectra and wavelet energy features," Knowledge-Based Systems, vol. 33, pp. 73-82, 2012.

[28] S. Wang, G. Ji, P. Phillips, and Z. Dong, "Application of genetic algorithm and kernel support vector machine to pathological brain detection in MRI Scanning," in Proceedings of the 2nd National Conference Information Technology Comp. Science, pp. 450-456, Shanghai, China, 2015.

[29] G. Yang, Y. Zhang, J. Yang et al., "Automated classification of brain images using wavelet-energy and biogeography-based optimization," Multimedia Tools and Applications, vol. 75, no. 23, pp. 1-17, 2015.

[30] Y. Zhang, G. Ji, J. Yang et al., "Preliminary research on abnormal brain detection by wavelet-energy and quantum-behaved PSO," Technology and Health Care, vol. 24, pp. S641-S649, 2016.

[31] Y.-D. Zhang, S. Chen, S.-H. Wang, J.-F. Yang, and P. Phillips, "Magnetic resonance brain image classification based on weighted-type fractional Fourier transform and nonparallel support vector machine," International Journal of Imaging Systems and Technology, vol. 25, no. 4, pp. 317-327, 2015.

[32] Y. Zhang, S. Wang, and L. Wu, "A novel method for magnetic resonance brain image classification based on adaptive chaotic PSO," Progress in Electromagnetics Research, vol. 109, pp. 325343, 2010.

[33] Y. Zhang, L. Wu, and S. Wang, "Magnetic resonance brain image classification by an improved artificial bee colony algorithm," Progress In Electromagnetics Research, vol. 130, pp. 369-388, 2012.

[34] S. Das, M. Chowdhury, and M. K. Kundu, "Brain MR image classification using multi-scale geometric analysis of ripplet," Progress in Electromagnetics Research, vol. 137, pp. 1-17, 2013.

[35] X. Zhou, S. Wang, W. Xu et al., "Detection of pathological brain in MRI scanning based wavelet-entropy and naïve Bayes classifier," in Proceedings of the International Conference on Bioinformatics and Biomedical Engineering, pp. 201-209, 2015.

[36] M. E. Tipping and C. M. Bishop, "Probabilistic principal component analysis," Journal of the Royal Statistical Society. Series B. Statistical Methodology, vol. 61, no. 3, pp. 611-622, 1999.

[37] S. Roweis, "EM algorithms for PCA and SPCA," in Advance in Neural Information Proccessing System, vol. 10, pp. 626-632, MIT Press, Cambridge, MA, USA, 1998.

[38] A. Ilin and T. Raiko, "Practical approaches to principal component analysis in the presence of missing values," Journal of Machine Learning Research, vol. 11, pp. 1957-2000, 2010. 

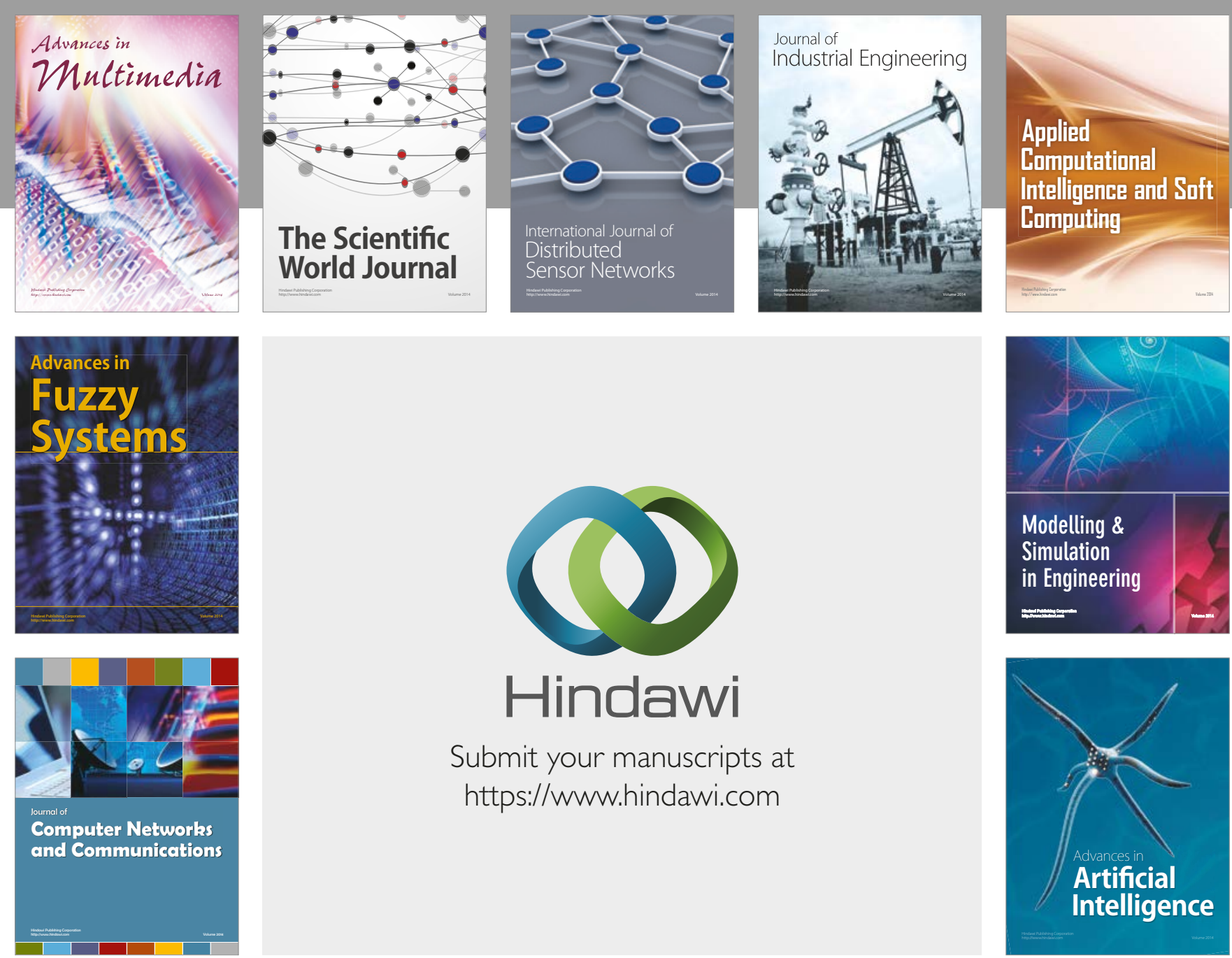

\section{Hindawi}

Submit your manuscripts at

https://www.hindawi.com
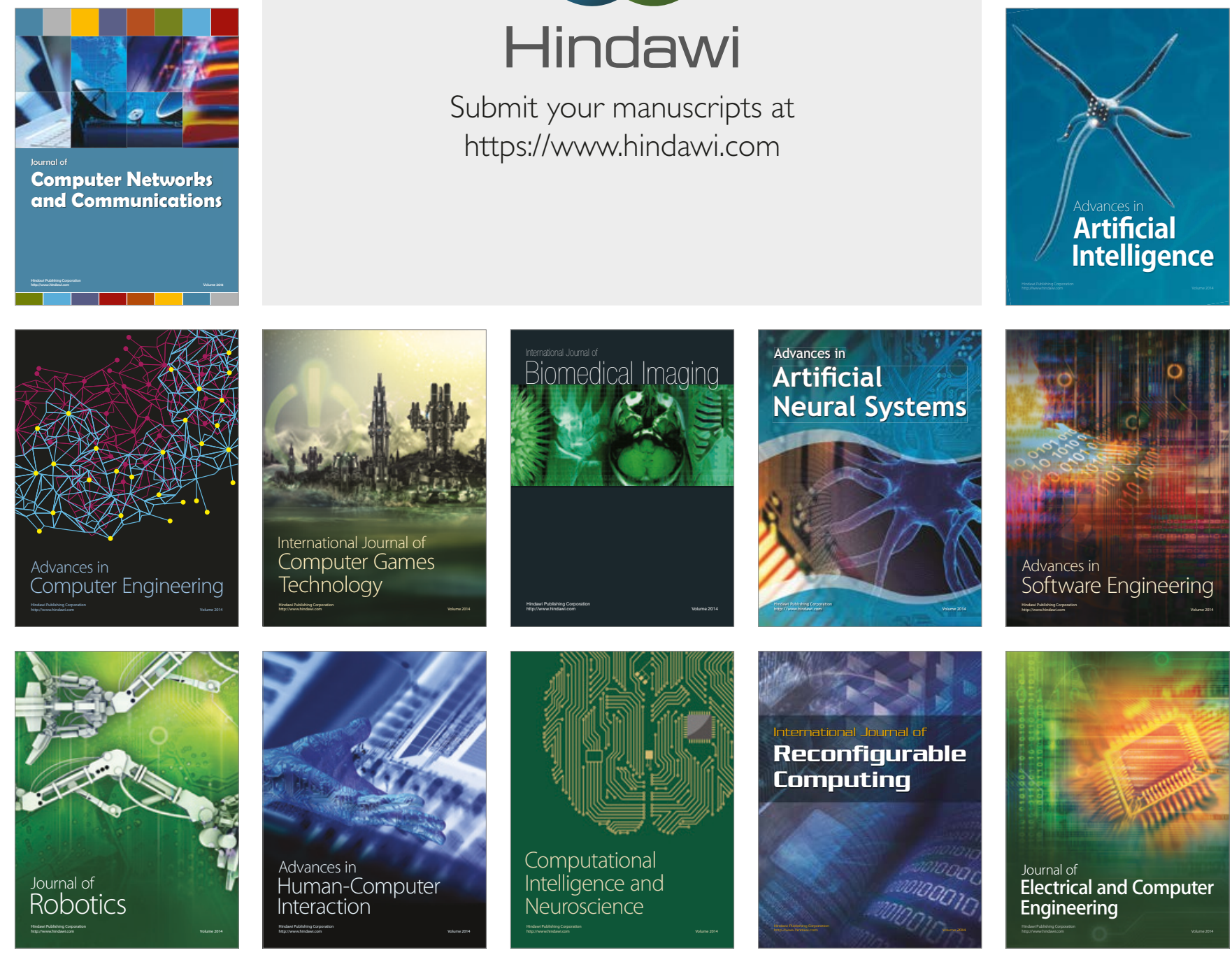